Editorial

\title{
CXCR4 Chemokine Receptor Overview: Biology, Pa- thology and Applications in Imaging and Therapy
}

\author{
Orit Jacobson $^{1 \bowtie}$, Ido Dov Weiss ${ }^{2}$ \\ 1. Hadassah Hebrew University Hospital, Cyclotron radiochemistry unit, Jerusalem, Israel. \\ 2. Hadassah Hebrew University Hospital, the Goldyne Savad Gene Therapy Institute, Jerusalem, Israel. \\ $\triangle$ Corresponding author: oritjacobson@yahoo.com
}

(c) Ivyspring International Publisher. This is an open-access article distributed under the terms of the Creative Commons License (http://creativecommons.org/ licenses/by-nc-nd/3.0/). Reproduction is permitted for personal, noncommercial use, provided that the article is in whole, unmodified, and properly cited.

Received: 2012.12.23; Accepted: 2012.12.24; Published: 2013.01.12

CXCR4 is a member of the chemokine receptor subfamily of seven transmembrane domained, G-protein coupled receptors, whose sole known natural ligand is CXCL12/SDF-1. CXCR4 is an unusual chemokine receptor by virtue of having expanded roles beyond leukocyte recruitment, including fundamental processes such as the development of the hematopoietic, cardiovascular, and nervous systems during embryogenesis. The receptor was first discovered as one of the co-receptors for HIV, and thereafter was also found to be expressed by multiple cancers including breast, prostate, lung, colon and multiple myeloma.

A number of recent studies have correlated high levels of CXCR4 expression in cancers with poor prognosis and with resistance to chemotherapy, in part through enhancing interactions between cancers and stroma. A possible role for CXCR4, and chemokine receptors generally, in cancer and metastasis was first suggested in studies of breast cancer, showing that the receptor plays a role in directing metastatic cells to CXCL12-expressing organs. Collectively, the data on CXCR4 in cancer suggest that this receptor increases tumor cell survival and/or growth and/or metastasis, making it a potentially attractive therapeutic target.

Due to the role of CXCR4 in HIV, multiple CXCR4 antagonists, although not sufficient for the treatment of HIV, are currently being evaluated and/or used for stem cell mobilization and as anti-tumor therapy. Some of the antagonists were also shown in animal models to be of use in evaluating
CXCR4 expression in whole tumors non-invasively by molecular imaging.

The research on CXCR4 has been ongoing for the last decade and yielded more than 7400 papers in PubMed as of December 2012. In this issue we tried to collect papers which represent CXCR4 involvement in various fields, from roles under normal conditions, to different pathologies, and finally to molecules targeting CXCR4 for theranostics.

The only CXCR4 antagonist which is currently approved for treatment of patients is Mozobil (also known as Plerixafor and AMD3100). This drug was approved by the FDA in December 2008 as an aid for mobilizing hematopoietic stem cells from the bone marrow to the bloodstream for collection and subsequent autologous transplantation in patients with non-Hodgkin's lymphoma and multiple myeloma. The drug approval was given as a combination therapy, with the more traditional stem cell mobilizer granulocyte-colony stimulating factor (GCSF). The role of CXCR4 in hematopoietic stem cell mobilization, and modulation of the receptor and its ligand CXCL12 (SDF-1) were discussed in the review by Ratajczak et al. [1].

The roles of CXCR4 and its ligand CXCL12 in developement are substantial, and are most evident from the fact that knock-out mice for either protein are lethal. We chose to bring an example of a less known role of CXCR4, which is its involvment in pancreatic developement. Recent findings in this area are reviewed by Katsumoto et al. in this issue [2].

The primary pathology in which CXCR4 was 
found to be essential is infection by human immunodeficiency virus (HIV), and this finding initiated vast research on the receptor. The role of CXCR4 by the T-trophic HIV as a co-receptor was later identified as a late phase of the disease, while the main co-receptor of the virus in the beginning of HIV infection is another chemokine receptor, CCR5. The detailed role of CXCR4 in HIV infection and the switch of the virus from CCR5-dependent M-trophic form to CXCR4-dependent T-trophic form is discussed by Vicenzi et al. [3].

As mentioned above, CXCR4 was found to play a significant role in tumor developement of both solid and blood cancers. Pre-clinical and clinical invstigations are being carried out in both cancer types using CXCR4 antagonists. we chose to bring examples of each cancer type: CXCR4 involvement in lung cancer solid tumor was review by Wald et al. [4], and the role of the receptor in acute myeloid leukemia (AML) was discussed by Peled et al. [5]. Both reviews seem to point CXCR4 as an important target for anti-cancer treatment, and help to treat patients of this pathology.

CXCR4 is expressed at different levels by all immune cells, and plays an homeostatic role in homing and retention of immune cells. However, under pathologic condition, such as inflammation and autoimmune disease, CXCR4 may exhibit other roles. Werner et al. discussed in this issue the role of CXCR4/CXCL12 axis in autoimmune disease, and the role of CXCR7 in regulating this axis as the second receptor for CXCL12, with a specific focus on inflammatory bowel disease [6].

To conclude this issue, we decided to include two additional reviews: one on the developement of small molecule inhibitors for CXCR4, which include Mozobil, as mentioned above, but also other molecules which show different pharmaceutical properties, reviewed by Debnath et al. [7]; the other review was written by us, discussing the utilization of different CXCR4 antagonists for positron emission tomography (PET) imaging, single photon emission computered tomography (SPECT) by radiolabeling the molecules with appropriate isotopes [8]. We have also included in the review some data on fluorescence imaging, although it is not currently widely used for human patients, it might become so in the future.

The collection of reviews presented in this special issue of Theranostics on CXCR4 will give the reader an idea of the various roles of this unique receptor in multiple areas of human biology, under both normal and pathologic conditions, and the recent advances that were acheived in targeting CXCR4 for theranostics.

\section{Competing Interests}

The authors have declared that no competing interest exists.

\section{References}

[1] Ratajczak MZ, Serwin K, Schneider G. Innate Immunity Derived Factors as External Modulators of the CXCL12 - CXCR4 Axis and Their Role in Stem Cell Homing and Mobilization. Theranostics 2013; 3(1):3-10. doi: $10.7150 /$ thno. 4621 .

[2] Katsumoto K, Kume S. The Role of CXCL12-CXCR4 Signaling Pathway in Pancreatic Development. Theranostics 2013; 3(1):11-17. doi: $10.7150 /$ thno.4806.

[3] Vicenzi E, Liò P, Poli G. The Puzzling Role of CXCR4 in Human Immunodeficiency Virus Infection. Theranostics 2013; 3(1):18-25. doi: $10.7150 /$ thno. 5392.

[4] Wald O, Shapira OM, Izhar U. CXCR4/CXCL12 Axis in Non Small Cell Lung Cancer (NSCLC) Pathologic Roles and Therapeutic Potential. Theranostics 2013; 3(1):26-33. doi:10.7150/thno.4922.

[5] Peled A, Tavor S. Role of CXCR4 in the Pathogenesis of Acute Myeloid Leukemia. Theranostics 2013; 3(1):34-39. doi:10.7150/thno.5150.

[6] Werner L, Guzner-Gur H, Dotan I. Involvement of CXCR4/CXCR7/CXCL12 Interactions in Inflammatory Bowel Disease. Theranostics 2013; 3(1):40-46. doi:10.7150/thno.5135.

[7] Debnath B, Xu S, Grande F, Garofalo A, Neamati N. Small Molecule Inhibitors of CXCR4. Theranostics 2013; 3(1):47-75. doi: $10.7150 /$ thno. 5376 .

[8] Weiss ID, Jacobson O. Molecular Imaging of Chemokine Receptor CXCR4. Theranostics 2013; 3(1):76-84. doi:10.7150/thno.4835. 